\title{
Subxiphoid uniportal approach is it just a trend or the future of VATS
}

\begin{abstract}
In recent years, Thoracic surgery has been developing very rapidly. This revolution in minimally invasive thoracic surgery is a small part of the major technological revolution in most medical fields. The ease of communication and access to information through the Internet, the publication of videos describing the operative techniques, the technological development in, high-resolution thoracoscopic cameras, and instruments designed specifically for this type of surgery have made it easier for surgeons to learn these techniques quickly and skillfully. A few years after the introduction to the technique of intercostal uniportal VATS as the least invasive technique in thoracic surgery, the subxiphoid uniportal VATS is now shown in the scene to present itself as a lesser invasive alternative. Does this technology really deserve to be an alternative or is it just a trend, and it will end soon. Should surgeons be satisfied with choosing to learn one technique or mixing the two techniques for the benefit to the patient and the surgeon.
\end{abstract}

Keywords: uniportal subxyphoid VATS, thoracic minimal invasive surgery, benefits of subxyphoid VATS, lung cancer
Volume 4 Issue 4 - 2017

\section{Firas Abu Akar}

Department of Cardiothoracic Surgery, Shaare Zedek Medical Center (SZMC), Israel

Correspondence: Firas Abu Akar, Department of Cardiothoracic Surgery, Shaare Zedek Medical Center (SZMC), Shmuel Bait St 12, Jerusalem, Israel, Email firasabuakar@gmail.com

Received: May 0I, 2017 | Published: May 26, 2017

\section{Discussion}

\section{Introduction}

The idea of using the thoracoscope to approach the surgical thoracic diseases is an idea that is not new at all. Jacobeas used the telescope to treat tuberculosis nearly 100 years ago. ${ }^{1}$ But techniques to this area have not developed much, and traditional surgery via open thoracotomy has remained the standard for more than seventy years. In the early 1990s, with the beginning with the development of minimally invasive surgeries, Roviero used the thoracoscope for the first complete pulmonary resection without resorting to conventional surgery, thus announcing the beginning of a new era in thoracic surgery. ${ }^{2}$ As a natural development within this field, attempts began to reduce the surgical trauma more and more, instead of four wounds to conduct the operation surgeons began to perform operations through three or two surgical incisions. ${ }^{3,4}$ Yamamoto was the first to think of using a single intercostal incision to perform simple thoracic operations with reporting a small series, ${ }^{5}$ followed by Rocco, who proved that this technique was feasible and safe ${ }^{6}$ Gonzalez's success in conducting the first anatomical resection through one intercostal incision opened doors for the use with this technique in other more complex procedures. ${ }^{7-9}$ To prevent damaging the intercostal nerves " which could happen no matter how large the wound is," some surgeons began to think of entering the chest cavity from other areas in the hope of getting rid of chronic pain after thoracic operations. In the beginnings, the subxiphoid incision was used as an auxiliary incision in the operations of anterior mediastinal masses' resection and thymectomies, ${ }^{10,11}$ until Liu and his colleagues proclaimed the first anatomical pulmonary resection using this technique. ${ }^{12}$ The question of which technique is best and safest for the patient still needs to be answered.

\section{Pre-operative preparing and patient selection}

In general, VATS procedure for an obese patient is difficult, especially through the subxiphoid approach. Apart from the difficulty of making the incision and finding the xiphoid cartilage, there is a great difficulty in entering the pleural cavity as this type of patients usually has a lot of pericardial fat that disrupts the process of access and consumes Lots of time for its removal. Thus, surgeons shouldchoose slim patients from the beginning of the learning curve for this type of operations.

\section{The incision}

As to where mentioned earlier, creating the incision in the subxiphoid procedures consumes more time than it's needed in the intercostal approach, especially in obese patients. In the subxiphoid operations, the destruction of any intercostal nerve is avoided, but usually, the surgeon needs to amputate the xiphoid cartilage in order to provide better exposure and to facilitate the instrumentation during the surgery.

\section{The exposure}

The exposure of the sagittal in the intercostal uniportal VATS is ideal and is the most similar minimal invasive view to the conventional open surgery "this may be one of the main reasons why this technique spreads rapidly and widely around the world."The exposure of the caudal view that we have in the subxiphoid approach has its advantages, especially for the removal of adhesions with the chest wall or with exposing the superior part of the thoracic cavity, in addition to the phenomenal bilateral panoramic view to both phrenic nerves in thymectomies and anterior mediastinal surgeries. On the other hand, there are many negatives, including the limited exposure of the posterior parts of the hilum and the limited vision during 
lymph node dissection (especially in Station 4 and Station 7) which is incomparable to the intercostal approach.

\section{Other important matters}

Subxiphoid approach may be very convenient for performing bilateral surgeries through one incision, anterior mediastinal surgeries or for the right upper and middle lobectomies, as the angle at which the staplers are introduced may be even more convenient than the intercostal approach to these lobes, but the limited vision of the lower lobectomies due to the presence of the diaphragm and compressing the heart during the left-side operations may make the subxiphoid technique not appropriate for the lower or left sided lobectomies.

\section{Conclusion}

Subxiphoid uniportal VATS approach is a new and interesting topic in the world of thoracic surgery. This technique has many advantages, in addition to the excellent exposure of in anterior mediastinal operations, avoiding the destruction, intercostal nerves and the possibility of entering the thoracic cavity from both sides through one incision are the strongest points of this technique. But at the same time, there are some negatives that we have to find solutions through scientific research and development of instruments. There may be a promising future in this type of surgery if we think about a way to integrate it with the robotic field.

\section{Acknowledgements}

None

\section{Conflict of interest}

The author declares no conflict of interest.

\section{References}

1. Jacobeus HC. Possibility of the use of the cystoscope for investigation of serous cavities. Munchen Med Wochenschr. 1910;57:2090-2092.
2. Roviaro G, Rebuffat C, Varoli F, et al. Videoendoscopic pul- monary lobectomy for cancer. Surg Laparosc Endosc. 1992;2(3):244-247.

3. McKenna RJ, Houck W, Fuller CB. Video-assisted thoracic surgery lobectomy: experience with 1,100 cases. Ann Thorac Surg. 2006;81(2):421-425.

4. Daniels LJ, Balderson SS, Onaitis MW, et al. Thoracoscopic lobectomy: a safe and effective strategy for patients with stage I lung cancer. Ann Thorac Surg. 2002;74(3):860-864.

5. Yamamoto H, Okada M, Takada M, et al. Video-assisted thoracic surgery through a single skin incision. Arch Surg. 1998;133:145-147.

6. Rocco G, Martucci N, La Manna C. Ten-year experience on 644 patients undergoing single-port (uniportal) video-assisted thoracoscopic surgery. Ann Thorac Surg. 2013;96:434-438.

7. Gonzalez D, Delgado M, Paradela M, et al. Uni-incisional video-assisted thoracoscopic left lower lobectomy in a patient with an incomplete fissure. Innovations. 2011;(Phila)6:45-47.

8. Gonzalez-Rivas D, Yang Y, Stupnik T, et al. Uniportal video-assisted thoracoscopic bronchovascular, tracheal and carinal sleeve resections. Eur J Cardiothorac Surg. 2016;49(1):16-16.

9. Abu Akar F, Gonzalez-Rivas D, Fink D. Management of bleeding from an abnormally located S6 arterial branch in a common origin with S2 branch during live surgery. J Vis Surg. 2016;2:87.

10. Zieliński M. Technique of transcervical-subxiphoid-VATS "maximal" thymectomy in treatment of myasthenia gravis. Przegla, $d$ Lekarski. 2000;57(5):64-65.

11. Kido T, Hazama K, Inoue Y, et al. Resection of anterior mediastinal masses through an infrasternal approach. Ann Thorac Surg. 1999;67(1):263-265.

12. Liu CC, Wang BY, Shih CS. ubxiphoid single-incision thoracoscopic left upper lobectomy. J Thorac Cardiovasc Surg. 2014;148:3250-3251. 